\title{
Tracking Football Player Movement From a Single Moving Camera Using Particle Filters
}

\author{
Anthony Dearden ${ }^{a}$, Yiannis Demiris ${ }^{a}$ and Oliver Grau $^{b}$ \\ ${ }^{a}$ Department of Electrical and Electronic Engineering \\ Imperial College London \\ Exhibition Road, London, SW7 2BT \\ E-mail: \{anthony.dearden, y.demiris\}@imperial.ac.uk \\ ${ }^{b}$ BBC Research, \\ Kingswood Warren, Tadworth, Surrey, KT20 6NP \\ oliver.grau@rd.bbc.co.uk
}

Keywords Soccer, Tracking, Particle Filter

\begin{abstract}
This paper deals with the problem of tracking football players in a football match using data from a single moving camera. Tracking footballers from a single video source is difficult: not only do the football players occlude each other, but they frequently enter and leave the camera's field of view, making initialisation and destruction of a player's tracking a difficult task. The system presented here uses particle filters to track players. The multiple state estimates used by a particle filter provide an elegant method for maintaining tracking of players following an occlusion. Automated tracking can be achieved by creating and stopping particle filters depending on the input player data.
\end{abstract}

\section{Introduction}

Tracking the movement of footballers in video footage has numerous uses for sportsmen and broadcasters alike. At the individual level, footballers can gain insight into their physiological performance [11]. At the group level, coaches can get information about the quality of the team's tactics, over the whole match or in particular set pieces. The broadcaster can use players' tracking information to provide enhanced statistical analysis and replays for the viewer. The BBC's Piero system ${ }^{1}$, for example, allows the visualisation of players and their path

\footnotetext{
${ }^{1}$ The Piero Project,

http://www.bbc.co.uk/rd/projects/virtual/piero/index.shtml
}

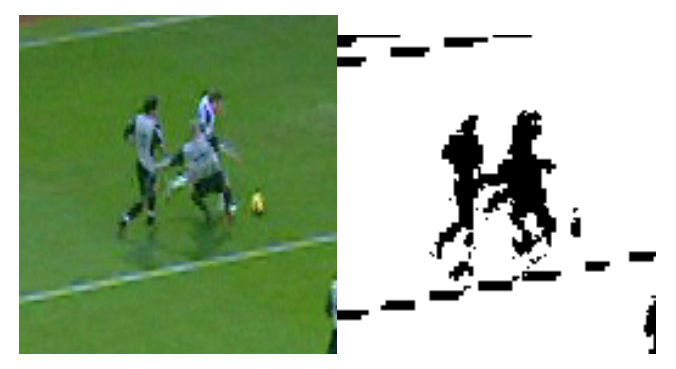

Figure 1: When players occlude each other, maintaining tracking can be difficult as the player region data (right) is ambiguous

in a 3D virtual stadium. The position of players in this system is currently determined by manually clicking over several frames of the action. A method that automatically extracts the exact positions of players would decrease the turn-around times of such a system. A further application would be automating $3 \mathrm{D}$ visualisation tools like the BBC's 'virtual replay' system².

This paper presents a system that enables players to be automatically tracked from a single, moving camera image. The alternative of electronic tracking has obvious acceptance problems with players and clubs [2]. Using an image-based approach has the further advantage that archived material can be analysed.

Tracking footballers in images is difficult because of occlusions; other players or even the referee can obscure the information about a tracked player, as shown in figure 1 . This is especially common during tackles, setpieces and action in front of the goal. Overcoming the

\footnotetext{
${ }^{2}$ http://news.bbc.co.uk/sport1/hi/football/default.stm
} 
problem of occlusions can be done by fusing data from multiple camera sources, with the idea that the ambiguity will not be present from all angles $[9,8]$. However, this adds to the complexity of the system; the goal here is to have a tracking system that can work directly from the image from a single moving camera tracking the action. Several techniques have been used previously to disambiguate player regions from a single camera source [5]. The first, also used here, is to apply morphological operators to erode close regions, hoping that they will split apart. Another method is to track the players using a graph representation, whereby the spatial relationship of players before a collision is stored so tracking can be continued when there is no longer an occlusion.

The main method used in this paper to maintain tracking of players through occlusions is by using a particle filter. Particle filters have become extremely popular in recent years as a method for Bayesian tracking without the restrictive assumptions of linearity and Gaussian distributions of a Kalman filter $[14,1]$. One aspect of particle filters which makes them especially useful in this situation is their ability to simultaneously maintain multiple hypotheses of the state of a tracked object.

The system presented here uses multiple particle filters to track multiple football players in a moving single video source. No assumptions are made here of the shape of the object being tracked: the player's shape is actually represented by the collection of particles. This point based model of shape has similarities with the point distribution model of [10]. Here the points represent a probabilistic distribution of the state of the player as opposed to image features of interest on a player. The first stage in the tracking, presented in section 2 involves using low-level computer vision techniques to extract player regions from the video data. This data is then used by a particle filter to estimate and maintain the position of the tracked player whilst he is in the image. The tracking can be initialised and stopped manually. Section 3 presents results of tracking, in particular for how the particle filter deals with occlusions. Conclusions, and areas of future work are discussed in section 4 .

\section{Tracking football player move- ment}

The input data for the tracking system is the continuous feed from the main 'spotter' camera situated in the stadium by the halfway line. This has numerous advantages over using broadcast football coverage as there are no scene changes, replays or overlayed graphics to contend with (although this information can be useful in itself for extracting information about the state of play e.g. [4]). The results presented here come from the FA Premier league game of Everton versus Blackburn Rovers from 2nd November 2005. Colour based tracking of the players for this game is especially difficult as the colours of the team are relatively similar - white with blue for Blackburn versus the grey strip of Everton. The image resolution is 720 by 576 at 25 frames per second. It is preprocessed with a de-interlacing filter, as the interlacing effects can degrade the player region data during fast movements.

As the field of view of the camera does not cover the entire pitch, the tracking system has to take into account that players will enter and leave the field of view. The camera is also prone to sudden fast movements as it tracks the action, making it harder to maintain the tracking of a player's position. As there is only one camera source used, there is no straightforward way of overcoming occlusions between players. The process of player tracking involves two steps: finding regions in the image where players are likely to be, and tracking players using these sources of information with a particle filter.

\subsection{Segmentation of player regions}

In order to track football players it is necessary to segment regions where players are likely to be from the rest of the image. Figure 2 gives an overview of the computer vision process used to extract player regions. The basic idea behind the process is to subtract the distinctive green colour of the pitch to leave regions which are likely to be players. This idea has been used on numerous previous occasions e.g. [13].

The colour of the pitch is represented as a one- or twodimensional histogram in HSV space. This histogram is back-projected onto each image and then with a threshold applied a binary pitch mask can be obtained. To estimate the entire pitch region, the pixels on the binary image are grouped into regions. By calculating the convex hull of the largest regions, the area in the image which the pitch covers can be calculated. Knowing the pitch regions in the image enables the tracking to be simplified by removing clutter from the crowd regions. The shape and position of the pitch region can also give information about the location on the pitch on which the camera is focused. As the colour of the pitch may drift over the duration of the match the histogram can be recursively updated by calculating the histogram of the pitch region excluding player regions. An obvious drawback of using the pitch to segment player regions is that it will fail when a player is off the pitch. However this only occurs at the very top of the pitch, usually when 


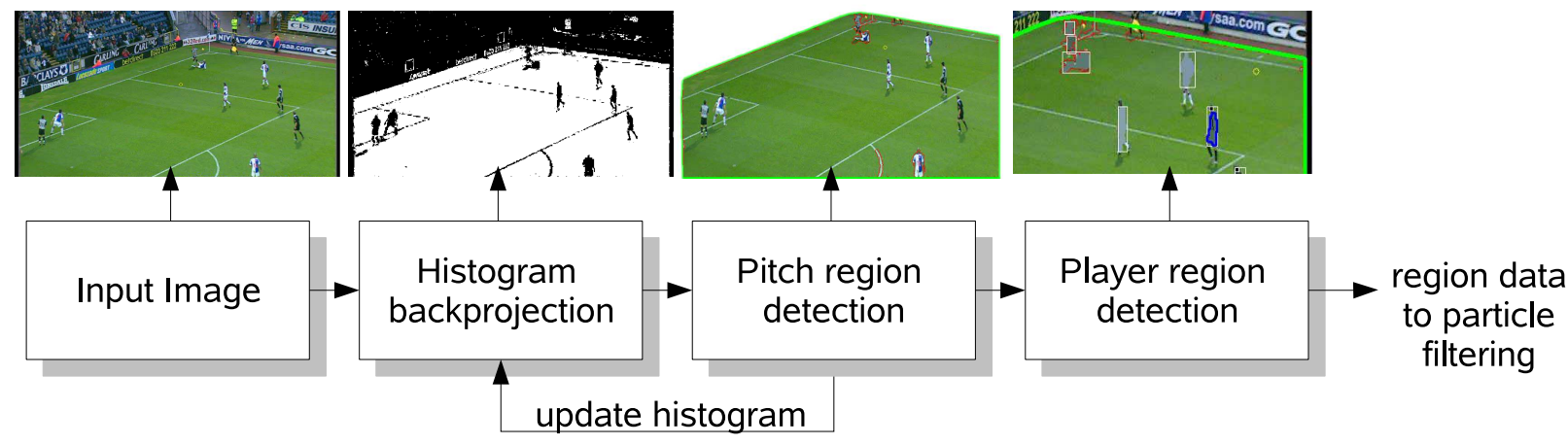

Figure 2: Overview of the region extraction process

a player is taking a throw-in. Furthermore, tracking a player taking a throw-in is a relatively low priority.

A binary image is now created of non-pitch colours within the pitch region showing possible player positions. To reduce the amount of clutter in this data, erosion and dilation morphological operators are applied. This works well at removing pitch markings from the mask, and reducing the likelihood of one player region occluding another. When the camera is highly zoomed out, however, the player regions can quite small (a few pixels wide). In this situation, erosion can potentially remove useful data. The player pixel regions can be grouped together to provide the raw data for the particle filter. Additional filtering of the data can be applied here to remove regions which are too large, small or wide to reasonably be a player region. The particle filter is well adapted to deal with clutter in the data because no assumptions are made about the type of probability distribution tracking the model. Following the segmentation, the observation data, $\mathbf{z}_{t}$, given to the particle filter is a set of $D$ regions, where each region, $\mathbf{C}$, is defined as:

$$
\mathbf{C}=(e, \mathbf{p}, \mathbf{c})
$$

where $e$ is a list of edge points on the region, $\mathbf{p}$ the centre of mass of the region and $\mathbf{c}$ the average colour of pixels contained within the region.

\section{$2.2 \quad$ Player tracking}

\subsubsection{Implementing an SIR particle filter}

The goal of any tracking system is to estimate the state of a system at time $t, \mathrm{x}_{\mathrm{t}}$, given a set of noisy observations up to this point, $\mathbf{z}_{1: t}$. The goal is therefore to estimate $p\left(\mathbf{x}_{\mathrm{t}} \mid \mathbf{z}_{1: \mathrm{t}}\right)$. If it is assumed that this is a first order Markov process, and we wish to estimate the state online, then the state estimation process can be split into two stages: prediction forward from the previous estimate followed by update with the new observation data [1]:

$$
\begin{gathered}
p\left(\mathbf{x}_{\mathrm{t}} \mid \mathbf{z}_{t-1}\right)=\int p\left(\mathbf{x}_{\mathrm{t}} \mid \mathbf{x}_{\mathrm{t}-1}\right) p\left(\mathbf{x}_{\mathrm{t}-1} \mid \mathbf{z}_{\mathrm{t}-1}\right) d \mathbf{x}_{\mathrm{t}} \\
p\left(\mathbf{x}_{\mathrm{t}} \mid \mathbf{z}_{t}\right)=\frac{p\left(\mathbf{z}_{\mathrm{t}} \mid \mathbf{x}_{t}\right) p\left(\mathbf{x}_{\mathrm{t}} \mid \mathbf{z}_{t-1}\right)}{p\left(\mathbf{z}_{\mathrm{t}} \mid \mathbf{z}_{t-1}\right)}
\end{gathered}
$$

Optimal solutions to these equations exist if the system is assumed to be linear in the form of a Kalman filter, with each conditional probability distribution being modeled as a Gaussian. However as figure 5shows the observation model here, $p\left(\mathbf{z}_{t} \mid \mathbf{x}_{t}\right)$, for example, is not Gaussian. Using a particle filter to track football players removes the necessity for the process to be modeled as linear and for the conditional probabilities to be modeled as Gaussians, and helps overcome the problem of tracking players through occlusions. A particle filter approximates a probability distribution with a weighted set of $N$ samples:

$$
p(\mathbf{x}) \approx \sum_{i=1}^{N} w^{i} \delta\left(\mathbf{x}-\mathbf{x}^{i}\right)
$$

with the condition that $\sum_{i=1}^{N} w^{i}=1$. The higher the number of particles, the closer the approximation, but the higher the computational load. One advantage of a particle filter is that it is an 'any time' algorithm - the number of particles and therefore the accuracy can be scaled according to the available resources. The type of particle filter used here is a sample importance resampling (SIR) particle filter [6], whose outline is shown in figure 3. The resampling stage is necessary because of particle degeneracy. Without this step, after a few iterations of the algorithm the weights of all but a few 


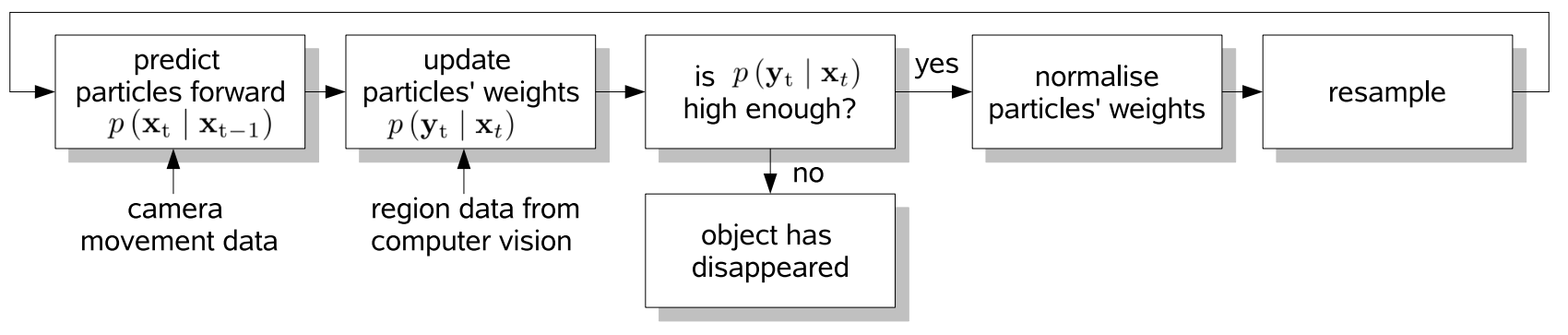

Figure 3: Overview of SIR particle filtering process

particle tend to towards zero, making them contribute little to the estimated state. More details can be found in [1].

\subsubsection{The player state}

The state tracked by the particle filter is defined as

$$
\mathbf{x}=(x, y, r, g, \dot{x}, \dot{y})
$$

where $x$ and $y$ are the position, $r$ and $g$ the chromacity values of this player and $\dot{x}, \dot{y}$ the velocity. A novel feature of the tracking performed here is that the state includes no information about the shape or size of the player being tracked, for example a bounding box width and height. The player's shape and size is actually represented by the collection of particles. The benefit of this is that no assumptions need to be made about the shape of the object being tracked, for example by approximating it to it bounding box. This improves the accuracy of the tracking, as no prior assumptions are made about the shape of the object being tracked. Furthermore, the size of the state space is reduced, meaning fewer particles are needed because the tracking is carried out in a lower dimension. However, to effectively represent a player's shape, a there is typically a minimum requirement of about 50 particles. An example distribution of a player's shape, $P\left(x_{t}, y_{t} \mid \mathbf{z}_{\mathbf{t}}\right)$ is shown in figure 4 . A possible future direction of work for player movement analysis involves extracting player posture information from this set of particles.

Implementing an SIR particle filter requires a model of the prior density, $p\left(\mathbf{x}_{\mathrm{t}} \mid \mathbf{x}_{\mathrm{t}-1}\right)$, to predict the state of a particle given its previous state and an observation model, $p\left(\mathbf{z}_{\mathrm{t}} \mid \mathbf{x}_{\mathrm{t}}\right)$, to update the weights of the particles given the observation data from the player segmentation section.

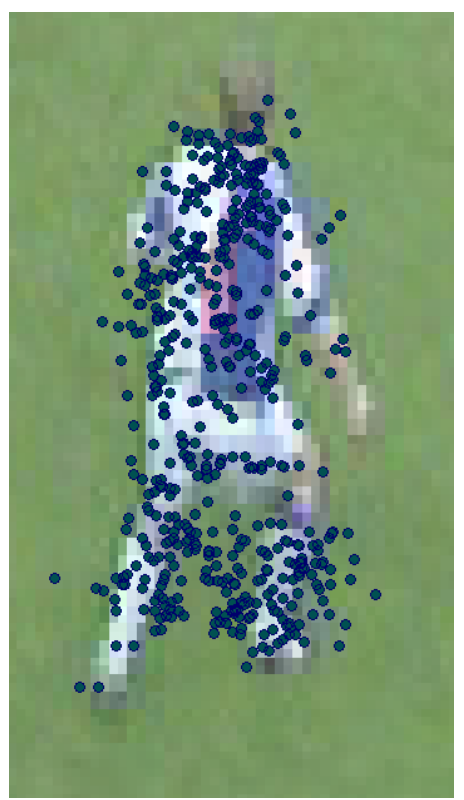

Figure 4: A set of 500 particles giving the probability distribution $P\left(x_{t}, y_{t} \mid \mathbf{y}_{\mathbf{t}}\right)$ of a player.

\subsubsection{The prior density}

Each particle's state $x_{\mathrm{t}}^{\mathrm{i}}$, is updated according the prior density.

$$
\mathbf{x}_{\mathrm{t}}^{\mathrm{i}} \sim p\left(\mathbf{x}_{\mathrm{t}} \mid \mathbf{x}_{\mathrm{t}-1}^{\mathrm{i}}\right)
$$

This is calculated separately for the position, colour and dynamic information in the state. Firstly, the position information, $x_{t}$ and $y_{t}$ is updated according to:

$$
\begin{aligned}
& p\left(x_{t} \mid \mathbf{x}_{t-1}\right) \propto x_{t}+\dot{x}_{t}+v_{x} \\
& p\left(y_{t} \mid \mathbf{x}_{t-1}\right) \propto y_{t}+\dot{y}_{t}+v_{y}
\end{aligned}
$$

where $v_{x} \sim N\left(0, \sigma_{x}\right)$ and $v_{y} \sim N\left(0, \sigma_{y}\right)$. The position transition standard deviations, $\sigma_{y}$ and $\sigma_{x}$, are set quite 
high to increase the spread of the particles $\left(\sigma_{x}=3.8\right.$, $\sigma_{y}=1.9$ ). This increases the search region of the particle filter for matching features in successive frames. $\sigma_{x}$ is set to be higher because camera movements in the $\mathrm{x}$ planes tend to faster and more unpredictable because of goal kicks and long-ball clearances.

The colours, $r_{t}$ and $g_{t}$, are updated according to:

$$
\begin{aligned}
& p\left(r_{t} \mid \mathbf{x}_{t-1}\right) \propto r_{t}+v_{r} \\
& p\left(g_{t} \mid \mathbf{x}_{t-1}\right) \propto g_{t}+v_{g}
\end{aligned}
$$

where $v_{r} \sim N\left(0, \sigma_{r}\right)$ and $v_{g} \sim N\left(0, \sigma_{g}\right)$. The colour transition standard deviation, $\sigma_{r}$ and $\sigma_{g}$, are set to be quite low $\left(\sigma_{r}=\sigma_{g}=0.001\right)$. This means that should a player be occluded by another, the individual particles will not 'forget' the colour of the original player they are tracking, and when the players separate again the particles should drift towards the correct player. As chromacity values are always between 0 and 1 , if the updated chromacity is outside this region it is re-sampled from a Gaussian distribution with mean of the average chromacity of all particles and standard deviation $\sigma_{r}$.

The velocity of the model, $\dot{x}_{t}$ and $\dot{y}_{t}$, is updated according to:

$$
p\left(\dot{x}_{t} \mid \mathbf{x}_{t-1}\right) \propto \gamma \dot{x}_{t-1}+(1-\gamma)\left(x_{t}-x_{t-1}\right)
$$

where $\gamma$ is an auto-regressive filtering parameter between 0 and 1 to smooth over a longer period the estimate of the player's velocity. For the results presented here $\gamma=0.6$. This approach is similar to the one taken in [3].

\subsubsection{The observation model}

The weight of each particle prior to resampling is calculated according to an observation model,

$$
w^{i} \propto p\left(\mathbf{z} \mid \mathbf{x}^{\mathbf{i}}\right)
$$

The time index, $t$, has been dropped here for the sake of equation readability. The basic effect of equation 12 should be to reward particles whose state maximises the likelihood of the observation data. To calculate the weight for a given particle $\mathbf{x}^{\mathbf{i}}$ and observation $\mathbf{z}$, firstly an error vector, $\mathbf{e}_{d}^{\mathbf{i}}$, is calculated for each of the $D$ regions, $\mathbf{C}_{d}$, where:

1. $\mathbf{e}_{\mathrm{x} d}^{\mathbf{i}}$ and $\mathbf{e}_{\mathrm{y}_{d}}^{\mathbf{i}}$ are set to the nearest distance of particle $\mathrm{x}^{\mathrm{i}}$ to the edge of region $\mathbf{C}_{d}$. If the particle is inside the region, $\mathbf{e}_{\mathrm{x} d}^{\mathbf{i}}$ and $\mathbf{e}_{\mathrm{y}_{d}}^{\mathbf{i}}$ are set to 0 .

2. $\mathbf{e}_{\mathrm{r}_{d}}^{\mathbf{i}}=\mathbf{C}_{\mathrm{r} d}-\mathbf{x}_{\mathrm{r}}^{\mathbf{i}}$
3. $\mathbf{e}_{\mathrm{g}_{d}}^{\mathrm{i}}=\mathrm{C}_{\mathrm{g}_{d}}-\mathbf{x}_{\mathrm{g}}^{\mathrm{i}}$

4. $\mathbf{e}_{\dot{\mathrm{x}} d}^{\mathbf{i}}=\mathbf{e}_{\dot{\mathrm{y}}_{\mathbf{d}}}^{\mathbf{i}}=0$

Finally, the weight of the particle is taken to be the sum of the Gaussians of the particle error vector with diagonal covariance vector $\mathbf{R}$ :

$$
w^{i}=\sum_{d=1}^{D} \exp \left(-\frac{\mathbf{e}_{\mathrm{d}}^{\mathbf{T}} \mathbf{R}^{-\mathbf{1}} \mathbf{e}_{\mathrm{d}}}{2}\right)
$$

The variation of this weight as a function of position for a particular scene and colour is shown in figure 5 .

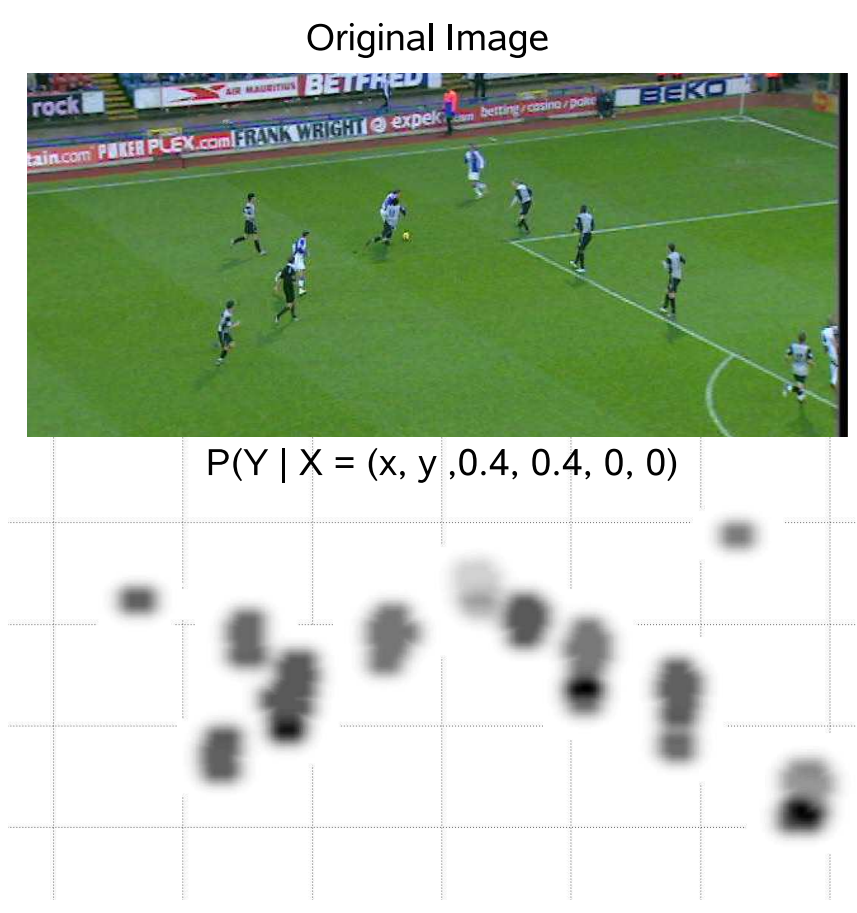

Figure 5: The posterior probability distribution function of observed data given the current state of a particle for each possible particle position. The darker the colour, the higher the probability $p\left(\mathbf{y}_{\mathrm{t}} \mid \mathbf{x}_{t}=x\right)$.

The covariance vector, $\mathbf{R}$, normalises the relative effect of each dimension of the state space on the final weight. If, for example the colour information is noisy, $\mathbf{R}_{\mathbf{r}}$ and $\mathbf{R}_{\mathrm{g}}$ can be increased to model this increased variance and reduce their effect on the weight. The calculation of the distance errors $\mathbf{e}_{\mathrm{x}_{d}}^{\mathbf{i}}$ and $\mathbf{e}_{\mathrm{y}_{d}}^{\mathbf{i}}$, is relatively expensive. It is therefore not performed for regions which are more than 100 pixels away from the particle, as the contribution to the weight from these regions is insignificant. 


\subsubsection{Detecting and removing tracked players}

Tracking players can be initialised manually, by selecting an initial region and creating a set of particles in this region as an estimate for $p\left(\mathbf{x}_{0}\right)$. A method for automatically tracking each player is to use any significantly large player region which does not contribute significantly towards an existing tracked model be used to initialise a new tracking region. The contribution of each region to existing models can be estimated from its contribution to the weights of particles in existing models:

$$
\text { contribution of region } d=\sum_{i=1}^{N} \exp \left(-\frac{\mathbf{e}_{\mathrm{d}}^{\mathrm{T}} \mathbf{R}^{-1} \mathbf{e}_{\mathrm{d}}}{2}\right)
$$

When a model has left the edge of the screen or pitch it can no longer be tracked, so the particle filter for this player can be stopped. There are two methods of doing this. The first, and most obvious way is to detect when a model is close to the edge of the pitch region calculated in section 2.1. A threshold of a few frames can be applied before the tracking is stopped completely should the player only briefly leave the field of view. A second, more general way, is to estimate the likelihood of model by summing the weights before resampling. This method could be useful for more general tracking situations outside of football matches where the object being tracked could leave the field of view from any position (e.g. a person exiting via a door).

\section{Results}

The final system runs at about 5fps on a $2.4 \mathrm{Ghz}$ Pentium. This is far from real time, but the current code is unoptimised and contains large amount output visualisation code. The particle filter itself uses about $5 \%$ of the entire processing resources with 200 particles - most of the processing power is therefore used by the computer vision data extraction process. Both the particle filter and computer vision code could be parallelised for modern multiple core CPUs.

Figure 6 shows the tracking of two players in $\mathrm{x}$-y-time space from a particular sequence using $\mathrm{N}=200$ particles per player. The sequence involves the two players occluding each other together with a third player and the referee, and one of the players briefly leaving the field of view. Only the mean of the probability distribution, $m \approx \sum_{i=1}^{N} w^{i} \mathrm{x}^{i}$ is shown. The centres of all the region data used to track are also shown. It can been seen that because the observation noise is modeled, the particle filter has a smoothing effect on the estimated trajectory of the players as a Kalman filter would. Figure 7 shows sample frames from the sequences, together with the particle distributions tracking the two players. The particle filters are able to maintain tracking of both players despite the occlusion occurring. As expected, when the occluding players separate again, the particles spread into multiple groups because of the increased uncertainty. The particles soon converge on the original player because the colour of the data is closer to the original player model.

A second tracking sequence for one player is shown in figure 8 . The player makes a run from the half way line to the box. On the way he is fully obscured on three occasions by the referee and other players. The particle filter is however able to maintain tracking the player after the occlusions. The tracking in this sequence eventually fails when the player leaves the green region of the pitch and can no longer be segmented.

Overall, the particle filtering approach to tracking works well at maintaining tracking of players involved in collisions, situations in which a Kalman filter would fair less well because of its inability to maintain multiple tracking hypothesis. The principal weakness of the current system is that, whilst multiple objects are tracked with multiple particle filters, no information is shared between the trackers meaning that occlusions involving players on the same can lead to the particle filters both tracking the same player after a collision. Vermaak uses multi-modal particle filter to overcome this problem, which involves clustering particles into the individual models being tracked, and normalising the weights over each model as opposed to the entire set as done here [14].

\section{Conclusions and future work}

A system was presented in which football players can be tracked on a pitch using an SIR particle filter. The non-linear and non-Gaussian nature of a particle filter enabled it to track players in spite of occlusions by multiple other players. It is hoped that soon ground truth tracking results can be obtained using simulated date from the BBC 'iview' project [7]. Future work will involve improving tracking reliability by including information from the tracking of all other players when updating each individual tracked player, as used in [14] or [12]. Currently the tracking is only done in image space. The tracking could be made more reliable by also tracking the movement of the camera and tracking the players in pitch space - this has been done by [12] within a particle filtering framework. The Such a real-time camera 


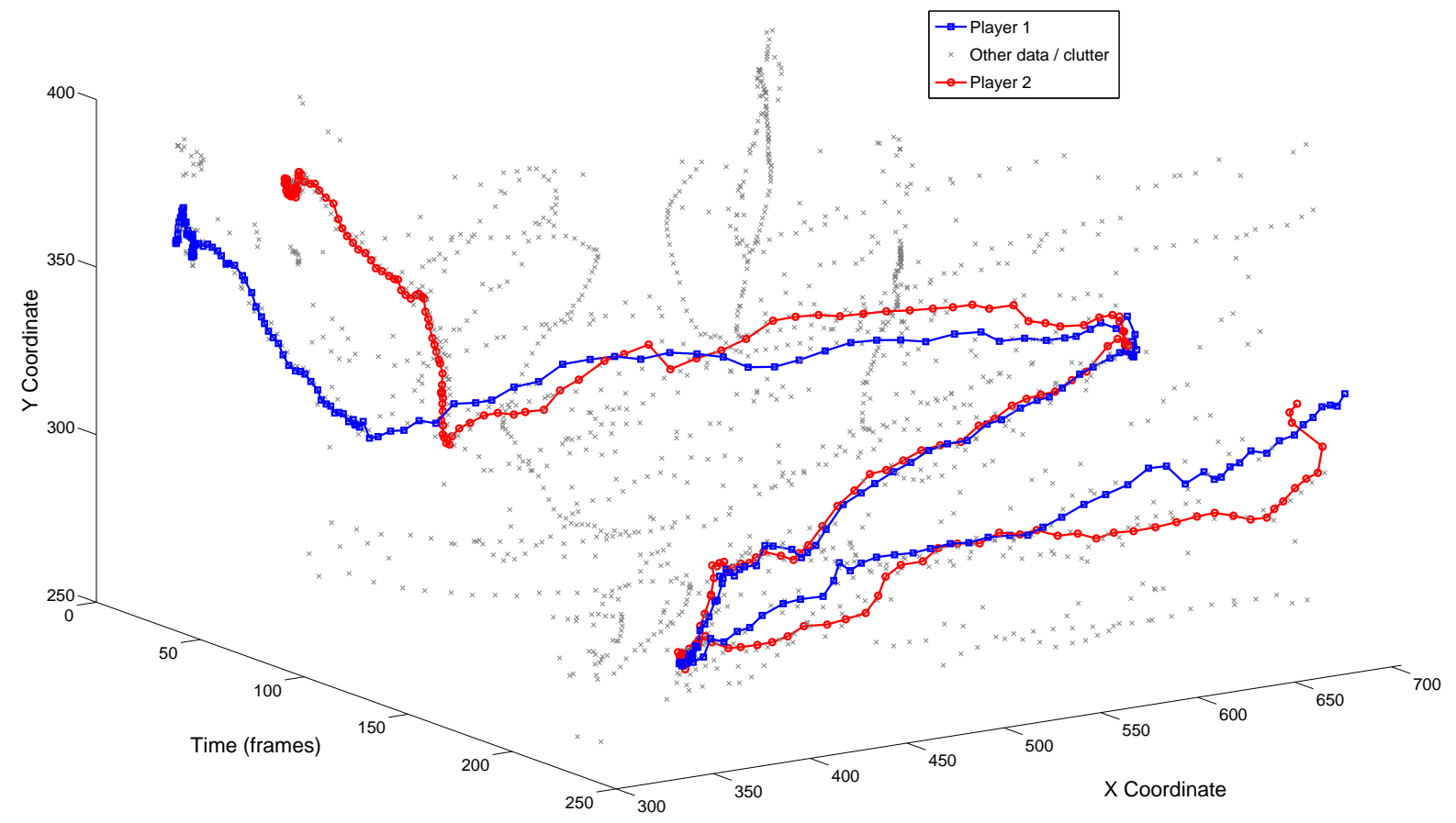

Figure 6: The tracking of two players involved in a tackle

tracking system is already being developed by BBC Research [7].

\section{Acknowledgments}

This work has been supported through a Doctoral Training Award from the UK's Engineering and Physical Sciences Research Council (EPSRC), and through a bursary from the BBC Research Department. The authors would like to thank the members of the BioART team at Imperial College and the Production Magic group at BBC Research and Development for their help and access to the video data.

\section{References}

[1] M. S. Arulampalam, S. Maskell, N. Gorden, and T. Clapp. A tutorial on particle filters for online nonlinear/non-gaussian bayesian tracking. IEEE transactions on signal processing, 50(2), 22002.
[2] Michael Beetz, Bernhard Kirchlechner, and Martin Lames. Computerized realtime analysis of football games. IEEE Pervasive Computing, 4:33-39, 2005.

[3] A. Blake and M. Isard. Active Contours. SpringerVerlag, 2nd edition, 2000.

[4] A. Ekin, A. Tekalp, and R. Mehrotra. Automatic soccer video analysis and summarization. In IEEE Trans. Image Processing, 2002.

[5] P. Figueroa, N. Leite, R. M. L. Barros, I. Cohen, and G. Medioni. Tracking soccer players using the graph representation. In Proceedings of the 17 th international conference on pattern recognition (ICPR04). Los Alamitos, Calif.; IEEE Computer Society, 2004.

[6] N. Gordon, D. Salmond, and A. F. M. Smith. Novel approach to non-linear and non-gaussian bayesian state estimation. In Proc. Inst. Elec. Eng., F, volume 140, pages 107-113, 1993. 


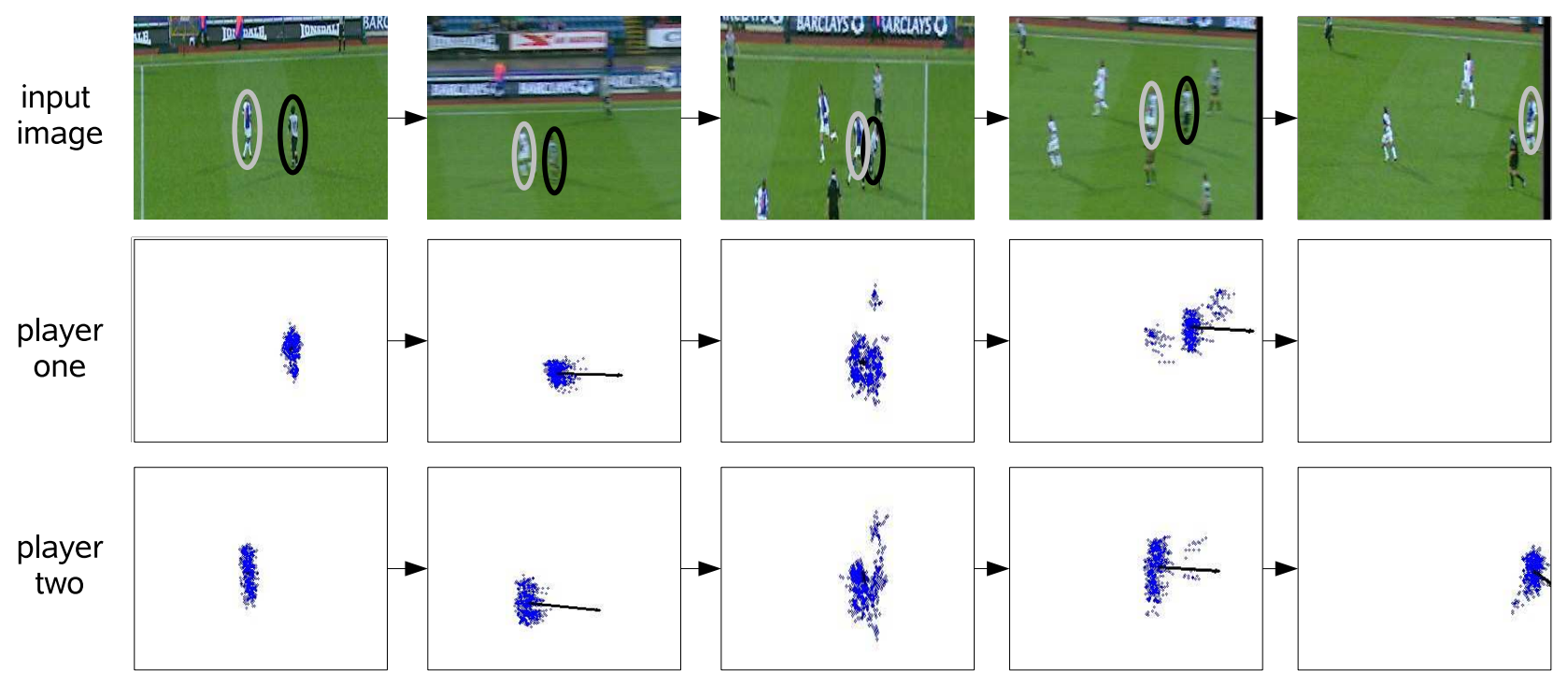

Figure 7: Frames from the tracking of two players. The last frame of player one is empty because the player has left the field of view. The black arrow in the particle represents the estimated velocity of the player. The players being tracked have been manually highlighted in the top images in black for player 1 and grey for player 2

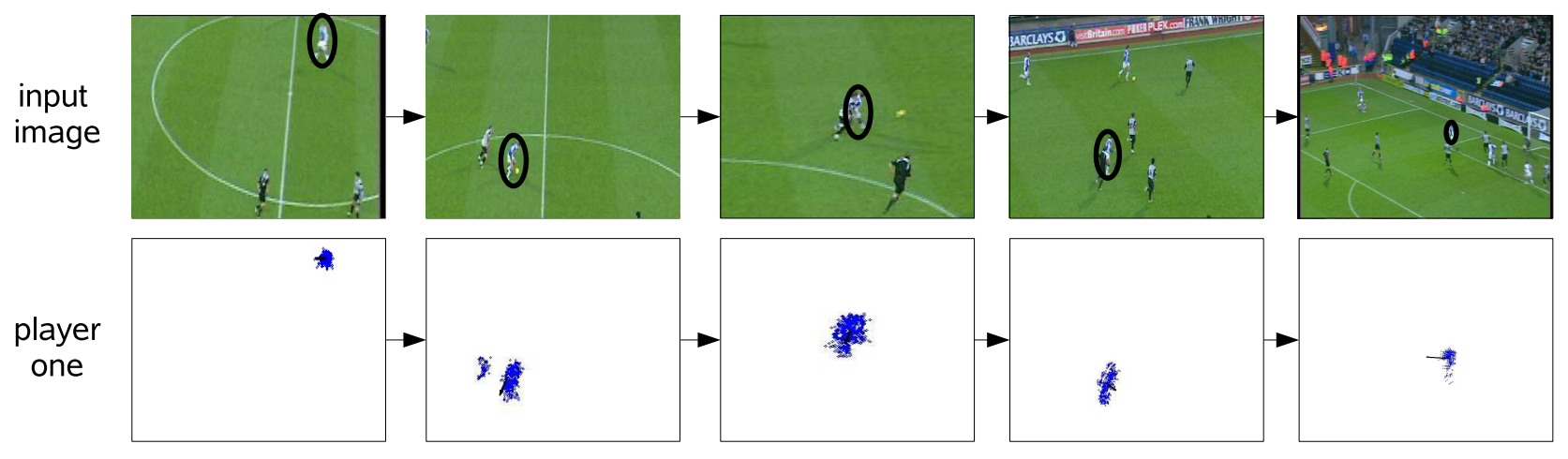

Figure 8: Selected close-ups of frames from tracking an occluded player making a run from the half way line. The player being tracked has been manually highlighted in the top images 
[7] Oliver Grau, M. Prior-Jones, and Graham A. Thomas. 3d modelling and rendering of studio and sport scenes for tv applications. In Proc. of WIAMIS 2006, 6th International Workshop on Image Analysis for Multimedia Interactive Services, April 2005.

[8] S. Iwase and H. Saito. Tracking soccer player using multiple views. In IAPR workshop on machine vision applications, 2002.

[9] S. Iwase and H. Saito. Tracking soccer players based on homography among multiple views. In Visual Communications and Image Processing, pages 283293, 2003.

[10] Tom Mathes and Justus H. Piater. Robust non-rigid object tracking using point distribution models. In British Machine Vision Conference 2005, 2005.

[11] P. G. O Donoghue, M. Boyd, J. Lawlor, and E. W. Bleakley. Time-motion analysis of elite, semiprofessional and amateur soccer competition. volume 41, pages 1-12. TEVIOT SCIENTIFIC, 2001.

[12] K. Okuma, A. Taleghani, N. de Freitas, J. Little, and D. Lowe. A boosted particle filter: Multitarget detection and tracking. In In Proc. European Conference Computer Vision, 2004.

[13] O. Utsumi, K. Miura, I. Ide, S. Sakai, and H. Tanaka. An object detection method for describing soccer games from video. In Multimedia and Expo, 2002. ICME '02. Proceedings. 2002 IEEE International Conference on. IEEE, 2002.

[14] J. Vermaak, A. Doucet, and P. Perez. Maintaining multi-modality through mixture tracking. In Ninth IEEE International Conference on Computer Vision (ICCV). IEEE Computer Society, 2003. 